“ (C) 2018 IEEE. Personal use of this material is permitted. Permission from IEEE must be obtained for all other uses, in any current or future media, including

reprinting/republishing this material for advertising or promotional purposes, creating new collective works, for resale or redistribution to servers or lists, or reuse of any copyrighted component of this work in other works." 


\title{
Decentralized Optimal Servo Control System for Implementing Instantaneous Reactive Power Sharing in Microgrids
}

\author{
Mohsen Eskandari, Li Li, Mohammad H. Moradi
}

\begin{abstract}
Active power is dispatched among distributed generation (DG) units in microgrids (MG) by means of $f / P$ droop control loop, which controls the frequency set-point of voltage source converter (VSI). Since the frequency is a global variable, active power sharing is implemented well proportional to droop coefficients. However, the reactive power is not shared accurately, through $V / Q$ control loop and according to the droop gains, as the voltage is a local variable. Furthermore, considering the small-scale of DG units, reactive power sharing should be implemented instantaneously to prevent DG units from overcurrent or even blackout of the MG. This paper deals with reactive power sharing issue in droop control-based MGs as well as stability and dynamic performance concerns of $V / Q$ control loop. A servo control system is designed to control power converters in MGs, by which droop-based VSIs are converted to servo VSIs (S-VSIs). A novel decentralized method is proposed to obtain the reactive power set-points of S-VSIs according to their droop coefficients and fuzzy particle swarm optimization method is used to optimize the S-VSI's parameters, so that, in addition to securing stability of the $V / Q$ loop, the desired (fast) response in reference tracking is achieved. The simulation results show that the proposed strategy is effective and its performance is not affected by delay or interruption of the existing low bandwidth communication link.
\end{abstract}

Index Terms-- Droop Control, Dynamic Stability, Microgrid (MG), Servo System, State Feedback Control, Power Converter, Virtual Impedance.

\section{INTRODUCTION}

$\mathrm{M}$ ICROGRID (MG) provides efficient energy management capability by clustering distributed generation (DG) units, i.e. renewable energy resources, micro-sources (MSs), and energy storage systems (ESSs), as well as reliability improvement by islanding operation capability [1]-[3]. MGs appear to be expanded greatly in the electrical distribution grids and play an important role in the implementation of smart grids. Presence of MGs in large numbers turns passive distribution grids into active bidirectional grids, which increases the influence of distribution grids on the overall stability of power networks. So utilizing an efficient control system in the MG is vital to perform the energy management while securing the stability of MG [4]-[8].

The droop-based control system, which mimics the behavior of synchronous machines in power sharing, has been proposed for power electronic-based MGs. In the conventional $f / P$ and $V / Q$ droop control, active power is controlled by frequency and reactive power is controlled by voltage. Frequency is a global variable, so active power is dispatched among DG units well based on the frequency drop and droop coefficients. On the other hand, implementing reactive power sharing among DG units with droop controller depends on the $V / Q$ droop coefficients. In this method, the voltage references, determined by droop controllers and inserted into the voltage source inverters (VSI) as input signals, are supposed to drop proportionally to droop gains. However, there are other voltage drops in the interconnecting power lines, which are not consistent with the droop controllers' behavior. This is because the voltage drop along the power line depends on the feeder impedance and power flow. So the feeder impedance mismatch or even different delivered power causes different voltage drop, which destroys accurate reactive power sharing [9]-[12]. This is why VSI is not a suitable tool for reference tracking in terms of reactive power sharing.

This issue is tackled in the considerable volume of studies in the area of MG control [11]-[20]; An improved droop control and a multifunctional droop controller are proposed in [13] and [14], respectively, for accurate reactive power sharing among DG units with equal rating. The strategy presented in [13] is based on injecting low bandwidth synchronization signal which modifies reference voltage to reduce the error in reactive power sharing. However, this leads to the voltage decline; although it is recovered by their proposed method, the power quality is influenced. In addition, this strategy is based on communication link, and the accuracy of power sharing among DG units with unequal ratings is not clarified. The accurate reactive power sharing is addressed in [14] by employing $V / \int Q . d t$ (instead of $V / Q$ ) control to embed reactive power historic information into droop control. In addition, $/ P . d t$ is added in the voltage droop equation to compensate the voltage deviation. However, ESSs are considered identical and the performance of the presented method is not evaluated for ESSs with various rating. Especially, voltage restoration by historic information of active power through $\int P . d t$ in the inductive dominant network appears to be not applicable for universal MG architectures, with various DG ratings and different power dispatch schedules. In addition, the system stability is not assessed. In [15] a voltage predictive controller is developed to generate a signal to the voltage reference to compensate the voltage deviation caused by droop controller. This method is of distributed scheme and a network estimator is needed to estimate the bus voltage. The estimator influences the performance of MG and stability analysis of the system is needed. To remove the effect of feeder impedance mismatch among DG units with equal size and to have accurate power sharing, a control strategy based on adaptive virtual impedance is presented in [16]. This method needs 
low-bandwidth communication link (LBWCL) to send the reference reactive power to each DG unit, and the system stability is not considered in adjusting virtual impedance.

Recently, consensus control (distributed averaging control) has become popular in the secondary control level in MGs. In [17] a consensus control is used to adaptively adjust the virtual impedance to achieve reactive power sharing and voltage regulation. In [18] a consensus-based voltage control is adopted to solve the reactive power sharing problem. In [19] a distributed averaging (consensus) secondary voltage controller is proposed to restore the voltage and frequency to their nominal values. Consensus control is actually based on communication link, and stability analysis is necessary. In addition, analysis of the flexibility of communication structure and interruption of communication link on the performance of the control system is a matter of concern. Furthermore, consensus control is located at the secondary level and its response speed is too slow to put in the primary level. A centralized secondary voltage control method is presented in [20] for multi-bus droop based MGs. Contribution of each DG unit in voltage regulation and reactive power compensation is optimally determined by the MG central controller (MGCC) considering MG efficiency, available capacity of DG units and power line limits. However, communication network is necessary to collect and transfer data between DG units and MGCC. In addition, the computation time for optimization process and communication delay are not discussed.

In addition to accuracy, quickness of establishing the precise reactive power sharing is important to prevent DG units from overcurrent, however, as the power converters have a low band current limitation. The problem in using consensus control [17]-[20], sampling and injecting synchronization signal [13], or computational-based [20] methods is the response time of the control system, which is in few seconds. Failing to share reactive power proportionally among DG units fast, may cause overcurrent relay trip or even the blackout of MG [21].

The contributions of this paper are outlined as:

1) A novel decentralized control strategy with fast response is proposed to address inaccurate reactive power sharing issues in MGs.

2) Voltage drop in the feeder impedance is modeled and accordingly converter's voltage reference is modified to compensate voltage drop in the feeder impedance. A novel decentralized method is presented to determine reactive power references according to $V / Q$ droop coefficients.

3) In order to develop a converter controller with reference tracking capability, a servo system-based control structure is proposed for VSI converters as servo-VSI (SVSI) to fast track the reactive power reference while it still provides voltage regulation as well.

4) An optimization-based method is proposed to determine the optimal S-VSI parameters, so that the desired control performance is achieved and the system stability via V/Q control loop is ensured. In the proposed method, virtual resistance and virtual inductance are considered as feedback gains and are optimized along with integrator gain by employing a pole-placement method. In this method, the system poles are moved to the desired location. The aim of pole-placement method adopted in the S-VSI is to yield the desired reference tracking response and secure the system stability. Because of insufficiency of conventional poleplacement methods (such as Linear-Quadratic-Gaussian design or Ackermann's formula) in the case of S-VSI, a fuzzy particle swarm optimization (F-PSO) method is developed to determine the feedback gain matrix.

5) In the proposed method, DG units do not have to be in equal size. In addition, the requirement of high bandwidth communication link is removed and the existing LBWCL is sufficient for proper operation of the proposed method. Furthermore, it is shown that the communication delay or even interruption of LBWCL does not affect the control performance.

The remainder of this paper is organized as follows: The MG topology is represented in Section II. In Section III, after discussing the drawback of the conventional droop control in accurate reactive power sharing implementation in MGs, the proposed method for reactive power reference determination is presented. After that, the design process of S-VSI is presented in Section IV. Then F-PSO method is developed to determine the optimum S-VSI parameters. Simulation results are presented in Section V to verify the effectiveness of the proposed control strategy. Finally, Section VI concludes the paper and gives some final remarks.

\section{Microgrid CONFIGURATION}

The MG topology is depicted in Fig. 1 where DG units are connected to the MG's bus, the point of common coupling (PCC), with different feeder impedances. The modeled feeder impedance includes converter's output LC filter, interconnecting power line and power transformer (if existing). Because of large impedance of the LC filter, the dominant feeder impedance is considered inductive. However, considerable resistance of the interconnecting power line makes the $\mathrm{X} / \mathrm{R}$ ratio of the feeder not as large as conventional power systems, and should be considered in the control system. MG is connected to the main grid at PCC via static circuit breakers which receives commands from the MG central controller (MGCC) for connecting and disconnecting purposes. In MG hierarchical control systems [8], LBWCL is needed to send set points from MGCC to the local controller of DG units and loads.

DG units in MGs are divided into two main categories: 1) dispatch-able units such as fuel-cells and micro (or gas) turbines which are responsible for load tracking, and 2) nondispatch-able units like photovoltaic cells and wind turbines which are operated at the maximum power tracking mode and unity power factor for the sake of efficiency improvement. Nevertheless, these DG units may be used as reactive power compensators whenever the sun light or wind power is unavailable. So it may be needed to send reactive power reference to them. It should be mentioned that non-dispatch-able units might become dispatch-able by installing a battery storage system on the DC side of the inverter.

Energy management strategy in connected mode $(\mathrm{CM})$ is that DG units produce active and reactive power according to the reference set points, which is sent by MGCC (economic dispatch), and the unbalance between production 
and consumption, because of errors in load and renewable generation forecasts, is compensated by the main grid. On the other hand, in islanded mode (IM) or in the transition from CM to IM, dispatch-able DG units are responsible to hold the balance between generation and consumption to keep the voltage and frequency within their permitted bands. Droop-based control system is implemented for power sharing which is proportional to droop gains. The droop gains are determined by MGCC according to the capacity of DG units to protect generation units from overloading and prevent circulating current among them [5].

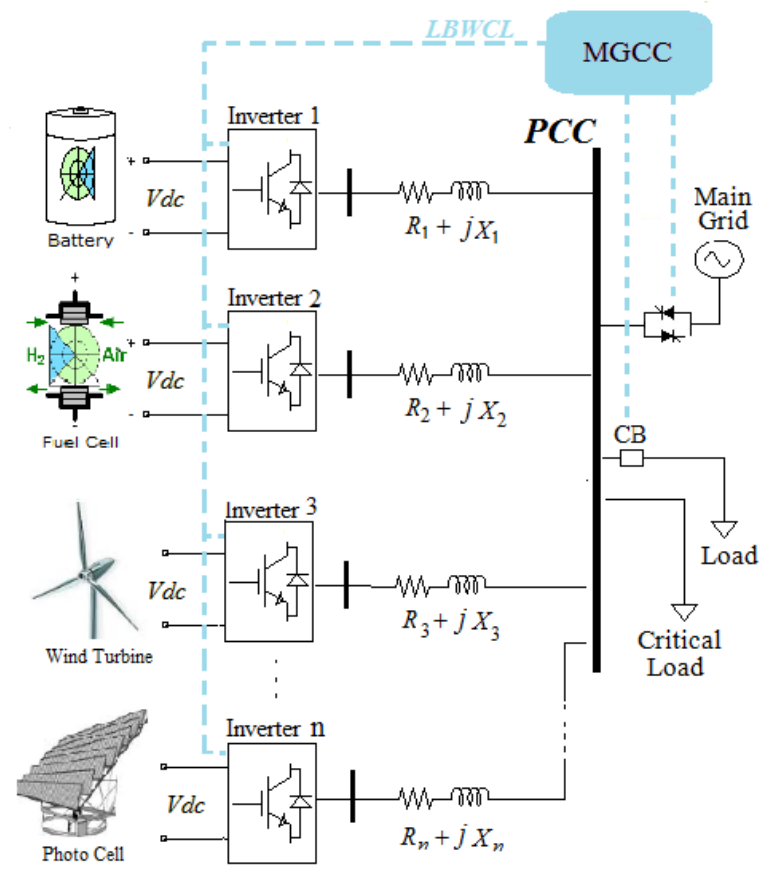

Fig. 1. MG configuration,

\section{REACTIVE POWER SHARING ISSUE}

The active power sharing is implemented well by the frequency droop control because dispatching active power among dispatch-able DG units depends on the frequency drop which is a global variable throughout the MG. However, the reactive power sharing is not the same case, which will be discussed in the next subsection. It should be kept in mind that, in the remainder of this paper, the presented method and discussions are related to IM operation of MGs and they are applicable for CM as well.

\section{A. Conventional Reactive Power Sharing}

Droop-based control methods have been used to implement active and reactive power sharing in MGs. The conventional $f / P$ and $V / Q$ control loops are established based on the following droop equations:

$$
\begin{aligned}
& w_{\text {ref }}=w^{*}-m\left(P-P^{*}\right) \\
& v_{o d, r e f}=V^{*}-n Q \quad \& \quad v_{o q, r e f}=0
\end{aligned}
$$

where $w_{\text {ref ref, }} v_{\text {od, ref }}$ and $v_{\text {oq, ref }}$ are radian frequency, direct and quadrature $(d-q)$ components of output voltage magnitude references, respectively, determined by droop control; $w^{*}$ and $V^{*}$ are nominal frequency (radian) and voltage magnitude, respectively; $P \& Q$ are the active and reactive power; $P^{*}$ is the active power reference which could be zero in the IM for the power sharing purpose; $m \& n$ are the $f / P$ and $V / Q$ droop coefficients.

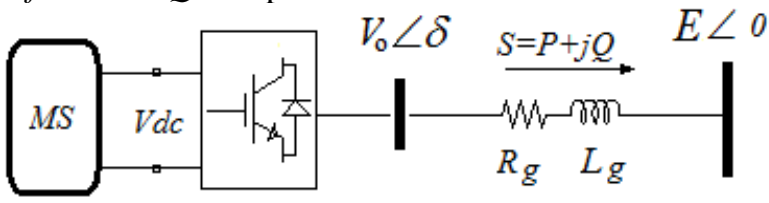

Fig. 2. A MS connected to the MG bus through a VSI and feeder

Fig. 2 shows a single MS connected to the bus via a VSI converter and interconnecting power line. Without loss of generality and for the sake of a better sense, it is supposed that the dominant impedance of the power line is inductive and the resistance is negligible. The power flow equations at the receiving end (PCC) [2] are:

$$
\begin{gathered}
P=\frac{V_{o} \cdot E \cdot \sin (\delta)}{X_{g}} \\
Q=\frac{V_{o} \cdot E-E^{2}}{X_{g}}
\end{gathered}
$$

where $V_{o}$ and $E$ are voltage magnitude at the sending and receiving ends of the feeder, respectively; $X_{g}$ is the feeder reactance and $\delta$ is the phase angle of the inverter's output voltage. Equation (4) can be rearranged as:

$$
V_{o}-\frac{X_{g}}{E} Q=E
$$

By embedding the droop control (2) into (5), it can be updated as:

$$
V^{*}-n \cdot Q-\frac{X_{g}}{E} Q=E
$$

Fig. 3 shows the voltage profile with respect to the reactive power variation based on (6). Ideally without considering the feeder impedance, the third term of left hand side of (6) is equal to zero. Then for the case of two DGs, the two equations for two DGs reach the equilibrium at bus voltage $E$ and the delivered reactive power is proportional to the droop gains; see the solid line labeled by a. By considering the feeder impedance, even with the same impedance for both DGs, two equations do not reach the equilibrium by delivering the determined reactive power; see the dashed lines labeled by $\mathbf{b 1} \boldsymbol{\&} \mathbf{b 2}$. Instead, the two equations reach the equilibrium at the voltage $E^{\prime}$, with an error in delivering specified reactive power; see the solid line label by c.

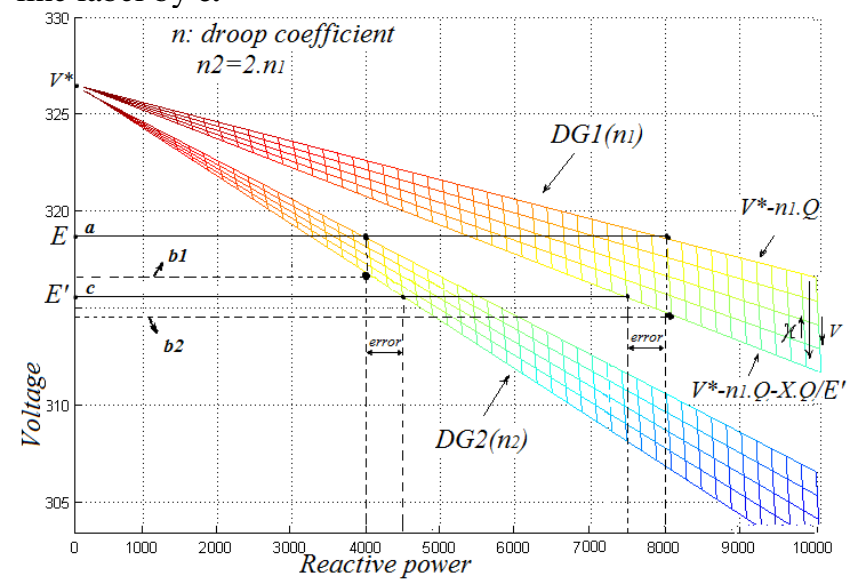

Fig. 3. Voltage profile with respect to the reactive power 


\section{B. Voltage Drop Modeling}

According to the previous subsection, the voltage drop over the feeder impedance is the obstacle of precise reactive power sharing. In order to estimate the voltage drop along the feeder, according to Park transformation for resistiveinductive lines, the voltage drop can be obtained as:

$v_{o d}-e_{d}=R_{g} \cdot i_{o d}-w^{*} \cdot L_{g} \cdot i_{o q}$

where $v_{o d}$ and $e_{d}$ are the direct component of the voltage at the sending (converter's output) and receiving ends (MG bus) of the feeder, respectively; $i_{o d}$ and $i_{o q}$ are the d-q components of the converter's output current flowing through the feeder, respectively; $R_{g}$ and $L_{g}$ are the resistance and inductance of the feeder, respectively. In a symmetric three-phase system, active and reactive powers are calculated as (8):

$P=e_{d} \cdot i_{o d} \quad, Q=-e_{d} \cdot i_{o q}$

By substituting $i_{o d}$ and $i_{o q}$ from (8) into (7), the voltage drop over the feeder impedance is estimated as in (9):

$v_{o d}-e_{d}=\frac{R_{g}}{e_{d}} \cdot P+\frac{w^{*} \cdot L_{g}}{e_{d}} \cdot Q$

The virtual impedance is modeled in the voltage reference as:

$v_{v i, d}=R_{v} \cdot i_{o d}-w^{*} \cdot L_{v} \cdot i_{o q}$

where $v_{v i, d}$ is the virtual impedance signal; $R_{v}$ and $L_{v}$ are the virtual resistance and inductance, respectively. The voltage profile in the feeder is obtained as:

$V^{*}-n \cdot Q-v_{v i, d}-\frac{\left(R_{g}\right)}{e_{d}} \cdot P-\frac{\left(w^{*}\left(L_{g}\right)\right)}{e_{d}} \cdot Q=e_{d}$

Two things are considered in developing (11) according to (9): a) Inserting the virtual impedance signal (10) into the voltage reference in (2), voltage reference is updated as $v_{\text {od, ref }}=V^{*}{ }_{-} n . Q-v_{v i, d}$. b) In the steady states, the voltage reference is followed by the output voltage of the converter $\left(v_{o d}=v_{o d, r e f}\right)$. With the same process as in (7)-(9), the voltage drop model of virtual impedance is developed as:

$v_{v i, d}=\frac{R_{v}}{e_{d}} \cdot P+\frac{w^{*} \cdot L_{v}}{e_{d}} \cdot Q$

So, taking the voltage drop model of virtual impedance in (12) into consideration, the voltage profile in (11) is updated as:

$V^{*}-n \cdot Q-\frac{\left(R_{g}+R_{v}\right)}{e_{d}} \cdot P-\frac{\left(w^{*}\left(L_{g}+L_{v}\right)\right)}{e_{d}} \cdot Q=e_{d}$

where the third and fourth terms are voltage drop model through the feeder impedance and virtual impedance. It is possible to modify the voltage reference in (2) as in (14), to remove the voltage drop from the voltage profile formula:

$v_{o d, r e f}=V^{*}-n \cdot Q-v_{v i, d}+\frac{\left(R_{g}+R_{v}\right)}{e_{d}} \cdot P+\frac{\left(w^{*}\left(L_{g}+L_{v}\right)\right)}{e_{d}} \cdot Q$

where the first and second terms on the right-hand side of (14) are the droop control model, the third term is virtual impedance signal in (10), and the fourth and fifth terms are included to compensate the voltage drop over the feeder impedance and virtual impedance in (13). Therefore, by applying (14) as control signal, the voltage drop along the feeder is obtained as $V^{*}-n \cdot Q=e_{d}$. More justification is that, in steady state, adding $R_{v}$ and $L_{v}$ in the fourth and fifth terms compensates voltage drop by virtual impedance caused by the third term (with different time constants to make both of them effective in dynamic response). Clearly, inserting $R_{g}$ and $L_{g}$ in (14) compensate voltage drop in the feeder.

\section{Reactive Power Reference Determination According to Q/V Droop Gains}

The voltage reference is modified as in (14) where the voltage drop over the feeder and virtual impedance is compensated. As a consequence, the voltage profile in the feeder (13) for the $i^{\text {th }}$ DG unit is:

$$
V^{*}-n_{i} \cdot Q_{i}=e_{d}
$$

where $n_{i}$ is the $V / Q$ droop coefficient of the $i^{t h} \mathrm{DG}$ unit. The MG bus voltage $\left(e_{d}\right)$ is sent to each DG unit by LBWCL (for the sake of preserving the band-width of communication link, $e_{d}$ could be sent to DG units in discrete form). Then at the local controller of each dispatch-able DG unit, the output reactive power of other dispatch-able DG units can be estimated by (15), and consequently the total reactive power can be obtained by (16).

$$
Q_{T}=\sum_{i=1}^{\beta} \frac{V^{*}-e_{d}}{n_{i}}
$$

where $\mathrm{Q}_{\mathrm{T}}$ is the total reactive power supplied by dispatchable DG units, $\beta$ is the number of dispatch-able DG units. Accordingly, the reference reactive power, $Q^{*}$, could be obtained for the $i^{\text {th }}$ DG units, which determines the contribution of the $i^{\text {th }}$ DG units in reactive power sharing:

$$
Q^{*}{ }_{i}=\alpha_{i} \cdot Q_{T}=\frac{1}{\sum_{z=1}^{\beta} \frac{n_{i}}{n_{z}}} \cdot Q_{T}
$$

where $Q_{i}{ }^{*}$ is the determined reference (set point) reactive power for the $i^{\text {th }} \mathrm{DG}$ unit, and $\alpha_{i}$ is the contribution of the $i^{\text {th }}$ DG units in reactive power sharing. The contribution of each dispatch-able DG units in reactive power sharing is obtained based on droop coefficients which, in turn, are specified by MGCC, according to (17), (18).

$$
\begin{aligned}
& n_{1} \cdot Q_{1}=n_{2} \cdot Q_{2}=\ldots=n_{i} \cdot Q_{i}=\ldots=n_{\beta} \cdot Q_{\beta} \\
& Q_{T}=Q_{1}+Q_{2}+\ldots+Q_{\beta}
\end{aligned}
$$

\section{PROPOSED SERVO-VSI}

In the previous section, reactive power reference is determined according to the droop gain. In this section, SVSI is proposed to fast track the reactive power reference to achieve instantaneous accurate reactive power sharing in MGs. In this section, the VSI model is presented first, and is followed by the design process of S-VSI.

\section{A. VSI Model}

The state-space model of the conventional droop-based VSI in Fig. 4 has been well developed in the literature [4]. The state space model of VSI is represented as:

$$
\begin{aligned}
& \dot{x}=A_{V S I} x+B_{V S I} \cdot u_{V S I} \\
& y=C_{V S I} x
\end{aligned}
$$

where $x \in R^{n}$ is the state vector of the plant, $u_{V S I} \in R^{m}$ is the control signal, $y \in R$ is the output signal, $A_{V S I} \in R^{n \times n}$ is the 
state matrix, $B_{V S I} \in R^{n \times m}$ is the input matrix, and $C_{V S I} \in R^{2 \times n}$ is the output matrix.

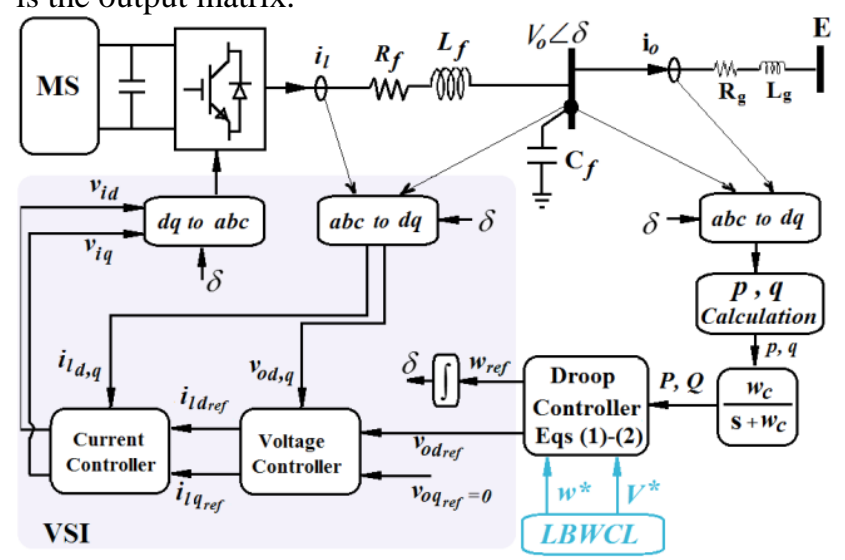

Fig. 4. Droop-based VSI control system

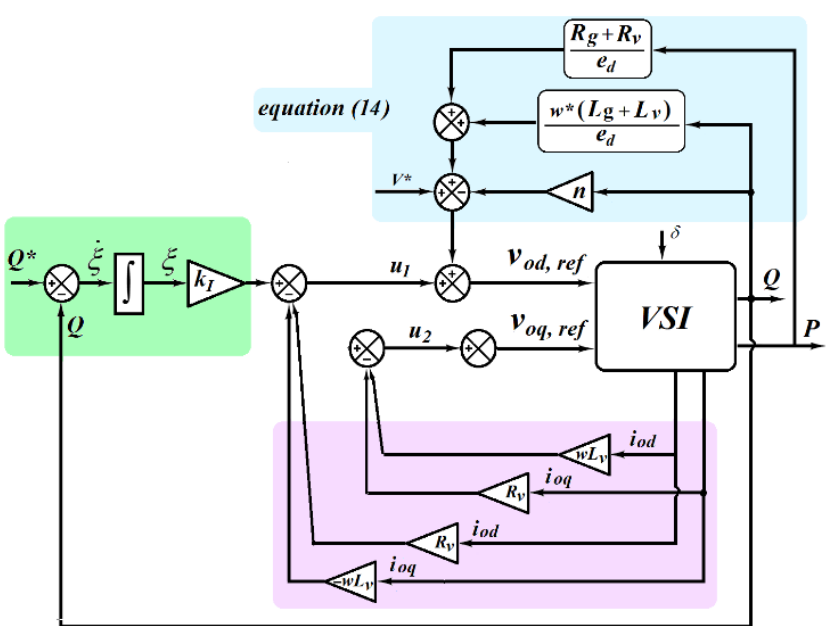

Fig. 5. Servo VSI (S-VSI)

The state variable matrix of VSI is:

$x=\left\lfloor\Delta \delta \Delta P \Delta Q \Delta \varphi_{d} \Delta \varphi_{q} \Delta \gamma_{d} \Delta \gamma_{q} \Delta i_{l_{d}} \Delta i_{l_{q}} \Delta v_{o_{d}} \Delta v_{o_{q}} \Delta i_{o_{d}} \Delta i_{o_{q}}\right\rfloor^{T}$

The state matrix $\left(A_{V S I}\right)$ is obtained from (A-1)-(A-10) as presented in the Appendix. The input and output matrices of VSI model are:

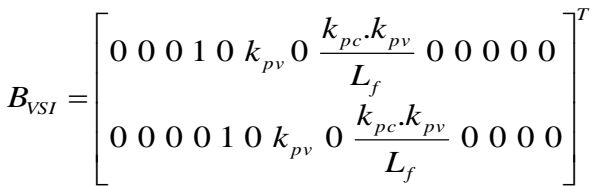

$$
\begin{aligned}
& C_{V S I}=\left[\begin{array}{l}
0100000000000 \\
0010000000000
\end{array}\right]
\end{aligned}
$$

The input and output signals are:

$u_{V S I}=\left[\begin{array}{c}\Delta v_{\text {od,ref }} \\ \Delta v_{\text {oq, ref }}\end{array}\right], y_{V S I}=\left[\begin{array}{c}\Delta P \\ \Delta Q\end{array}\right]$

\section{B. Servo-VSI}

The $V / Q$ control loop of the proposed S-VSI is illustrated in Fig. 5, which is used instead of the droop controller box in Fig. 4(a) (the $f / P$ control loop is ignored). For the sake of reference tracking purpose, an integrator is inserted in the feedforward path between the error comparator, of the given output variable $(Q)$ and its reference set point $\left(Q^{*}\right)$, and the plant. The integrator output $(\xi)$ is added to the voltage reference, the green box in Fig. 5, after multiplying the integrator gain $\left(k_{I}\right)$. The reference reactive power $\left(Q^{*}\right)$ is the reference input signal to the S-VSI, determined by MGCC in the CM, and by our proposed method in the IM as presented in the Section II.C.

The feedback loops, represented as the blue box in Fig. 5, are embedded into the control system to compensate the voltage drop over the feeder by modifying the voltage references according to (14). The dynamic version of (14) is obtained as:

$\left[\begin{array}{l}\Delta v_{\text {od, ref }} \\ \Delta v_{\text {oq, ref }}\end{array}\right]=\left[\begin{array}{c}\Delta u_{1} \\ \Delta u_{2}\end{array}\right]+\left[\begin{array}{cc}\frac{R_{g}+R_{v}}{e_{d}} & \frac{w^{*}\left(L_{g}+L_{v}\right)}{e_{d}}-n \\ 0 & 0\end{array}\right]\left[\begin{array}{l}\Delta P \\ \Delta Q\end{array}\right]$

Consequently, the input signal of VSI is updated as:

$$
\begin{aligned}
u_{V S I}=\left[\begin{array}{c}
\Delta v_{o d, r e f} \\
\Delta v_{o q, r e f}
\end{array}\right]= & u_{S-V S I}+M_{d r o o p} \cdot y_{V S I} \\
& =u_{S-V S I}+M_{d r o o p} \cdot C_{V S I} \cdot x
\end{aligned}
$$

where $u_{S-V S I}=\left[\begin{array}{lll}\Delta u_{1} & \Delta u_{2}\end{array}\right]^{\mathrm{T}}$ as in (27)-(28):

$$
\begin{aligned}
& \Delta u_{1}=-R_{v} \cdot \Delta i_{o_{d}}-\left(-w^{*} \cdot L_{v}\right) \cdot \Delta i_{o_{q}}+k_{I} \cdot \xi \\
& \Delta u_{2}=-R_{v} \cdot \Delta i_{o q}-\left(w^{*} \cdot L_{v}\right) \cdot \Delta i_{o_{d}}
\end{aligned}
$$

where $R_{v}, L_{v}$ are the virtual resistance and virtual inductance (the virtual impedance feedback loops is highlighted by pink in Fig. 5); $k_{I}$ is the feedforward integrator gain; and $\xi \epsilon$ $R$ is the integrator output, the feedforwarded signal, defined as:

$$
\xi=\int\left(Q^{*}-Q\right) d t
$$

According to Fig. 5, the S-VSI output is:

$$
\begin{aligned}
y_{S-V S I} & =Q=\left[\begin{array}{ll}
0 & 1
\end{array}\right] C_{V S I} \cdot x \\
& =C_{S-V S I} \cdot x
\end{aligned}
$$

From (29), the new state variable is defined as:

$$
\dot{\xi}=Q^{*}-y_{S-V S I}=Q^{*}-C_{S-V S I} x
$$

Therefore, the augmented state space model is:

$$
\dot{\hat{x}}=A_{S-V S I} \cdot \hat{x}+B_{S-V S I} \cdot u_{S-V S I}
$$

where

$$
\begin{aligned}
& \hat{x}=\left[\begin{array}{l}
x \\
\xi
\end{array}\right] \\
& A_{S-V S I}=\left[\begin{array}{rr}
A_{V S I}+B_{V S I} M_{\text {droop }} C_{V S I} & 0 \\
-C_{S-V S I} & 0
\end{array}\right] \\
& B_{S-V S I}=\left[\begin{array}{c}
B_{V S I} \\
0
\end{array}\right] \\
& u_{S-V S I}=-K . \hat{x}
\end{aligned}
$$

Here $\mathrm{K}$ is the state feedback gain matrix as:

$$
K=\left[\begin{array}{cccc}
0_{1 \times 11} & R_{v} & -w \cdot L_{v} & -k_{I} \\
0_{1 \times 11} & w . L_{v} & R_{v} & 0
\end{array}\right]
$$

Finally, by combining (33)-(37), the closed-loop S-VSI state equation is obtained as: 


$$
\dot{\hat{x}}=\left(A_{S-V S I}-B_{S-V S I} \cdot K\right) \hat{x}
$$

Highlight 1: Virtual impedance is considered for decoupling the active and reactive power control [22]-[24]. In the proposed S-VSI, the virtual impedance is adopted as the state feedback gain, which will be optimally determined to stabilize the $V / Q$ loop. The drawbacks of virtual impedance include limiting maximum transferable power and voltage variation band, which are resolved by adding the feedforward input signal coming from the integrator and also by modeling the voltage drop in the reference voltage; see (14). It should be noticed that the time constants of two feedback loops in Fig. 5 are different from each other. Therefore, both loops are effective in the system dynamic response.

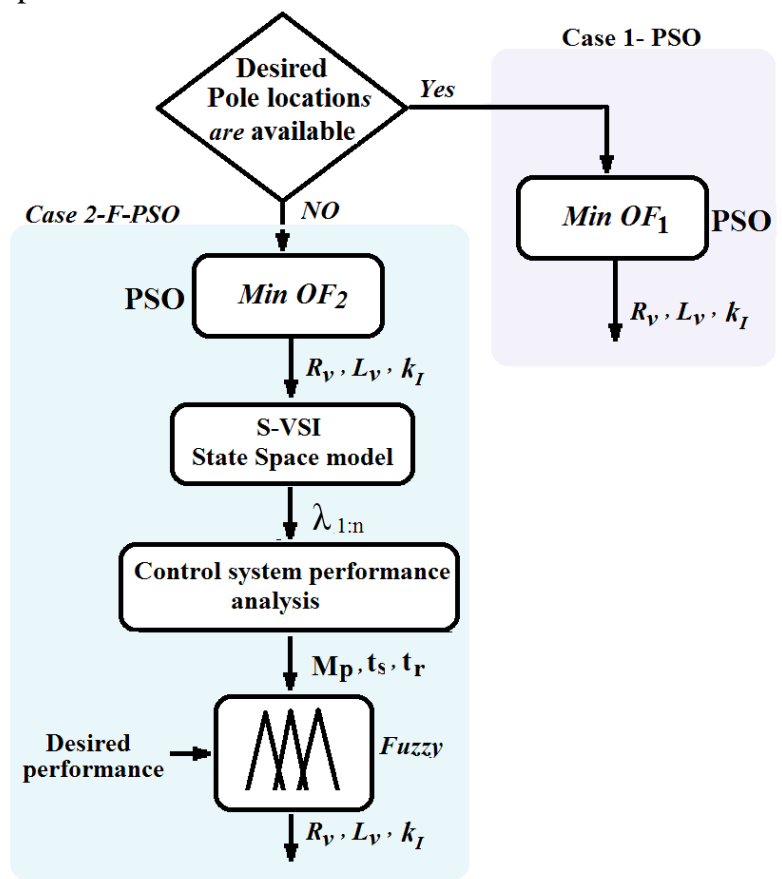

Fig. 6. Optimization flowchart

\section{Optimum S-VSI}

The equivalent state matrix of S-VSI is obtained as in (38). The next step is to optimally determine $R_{v}, L_{v}$ and $k_{I}$ to achieve the suitable control performance in reference tracking as well as ensure the stability of $V / Q$ control loop.

Highlight 2: There are two goals in the design of S-VSI:

i) Reactive power sharing: the integrator is embedded in the feedforward path, and the virtual impedance is adopted to decouple $f / P$ and $V / Q$ control loops,

ii) Stabilizing $V / Q$ control loop with good dynamic performance: In the state-space design of servo systems, state feedback gain matrix can be determined based on the pole placement (allocation of system poles to the desired locations) to achieve the desired control performance and system stability. The S-VSI is constituted by considering virtual impedance gains as state feedback gains to employ pole-placement method and tuning the virtual impedance and integrator gain.

In conventional pole placement methods such as LinearQuadratic-Gaussian design and Ackermann's formula, it is needed that all state variables are adopted in the feedback (state feedback), while in the S-VSI, there are two original states and one integrator, that is, three extended states in the feedback (output feedback). Some methods, e. g. linear matrix inequality (LMI), have been proposed to solve output feedback stabilization problems [25]-[27]. However, the computational time for solving these methods is too long. Besides, only the system stability is guaranteed while the dynamic performance is not taken into account. To this end, an optimization algorithm is proposed to optimally determine the virtual impedance and integrator gain, so that the best system performance and stability of the control loop are achieved.

\section{1) Objective Function:}

Case 1. If the desired pole locations (to achieve the desired reference tracking response as well as dynamic stability) are known in advance, considering that the system is controllable, the dominant poles could be easily moved to the known locations by optimizing the following objective function (39) using PSO:

Min $O F_{1}=\sum_{i=1}^{n} a b s\left(e i g(S-V S I)_{i}-e i g(D S)_{i}\right)$

where $a b s$ denotes the absolute value, eig $(S-V S I)$ is the eigenvalues of S-VSI state-space model, eig $(D S)$ is the desired system eigenvalues, and $n$ is the number of states which is 14 for our S-VSI.

Case 2. If the desired poles are not known in advance, the following objective function (40) is optimized using F-PSO:

$\operatorname{Min} O F_{2}=\sum_{i=1}^{n}\left(\begin{array}{c}\operatorname{real}\left(\text { eig }(S-V S I)_{i}\right) \\ + \text { abs }\left(\text { imaginary }\left(\text { eig }(S-V S I)_{i}\right)\right)\end{array}\right)$

The flowchart diagram of the optimization process is presented in Fig. 6.

\section{2) Constraints:}

The lower and upper limits of the optimizing variables are defined based on the minimum requirement to decouple the active and reactive power control (increase $X / R$ ratio) for the virtual impedance $\left(R_{v} \& L_{v}\right)$ and the voltage magnitude limit for integrator gain $\left(k_{I}\right)$ :

$$
\begin{aligned}
& L_{v, \text { min }} \leq L_{v} \leq L_{v, \text { max }} \\
& R_{v, \text { min }} \leq R_{v} \leq R_{v, \text { max }} \\
& k_{I, \min } \leq k_{I} \leq k_{I, \max } \\
& \forall i \text { real }\left(\operatorname{eig}(S-V S I)_{i}\right) \leq 0
\end{aligned}
$$

\section{Tips to Improve Control System Dynamics, Accuracy and Reliability:}

Tip\#1. After reaching the steady state, the linearized state-space model of the system is updated around the operating point, and the virtual impedance $\left(R_{v} \& L_{v}\right)$ and integrator gain $\left(k_{I}\right)$ is modified by optimizing the objective function to secure the dynamic performance of the control system.

Tip\#2. Voltage drop over the feeder impedance is modeled in the voltage reference as in (14) so that (15) is obtained. The other factor influencing the output voltage of S-VSI is the feedforward integrator effect $\left(\mathrm{k}_{\mathrm{I}}, \xi\right)$, which should be modeled in (15) to estimate the reactive power of other dispatch-able DG units accurately. So in the steady 
states, the value of $V^{*}$, which is same for all S-VSIs, is updated using (42) for each individual unit as $V^{*}$, and then is sent to other S-VSIs by LBWCL to estimate the reactive power delivered by other DG units in the next transient and improve the accuracy of the control system;

$$
V_{i}^{*}=V^{*}+k_{I_{i}} \cdot \xi_{i}=V^{*}+k_{I_{i}} \cdot \int\left(Q_{i}^{*}-Q_{i}\right) d t
$$

where $V_{i}^{*}$ and $k_{I i}$ are the nominal voltage and integrator gain at the $i^{\text {th }} \mathrm{S}$-VSI, respectively.

Tip\#3. Whenever $e_{d}$ is unavailable, because of LPWCL interruption, it could be obtained from (43) to improve the reliability of the control system.

$$
e_{d}=V_{i}^{*}-n_{i} \cdot Q_{i}
$$

As the mentioned processes in Tips $1 \& 2$ are done in the steady state, there is no concern about the delay and bandwidth of the communication link and the idea in Tip 3 removes the concern about the interruption of communication link. The flowchart of the proposed method is given in Fig. 7.

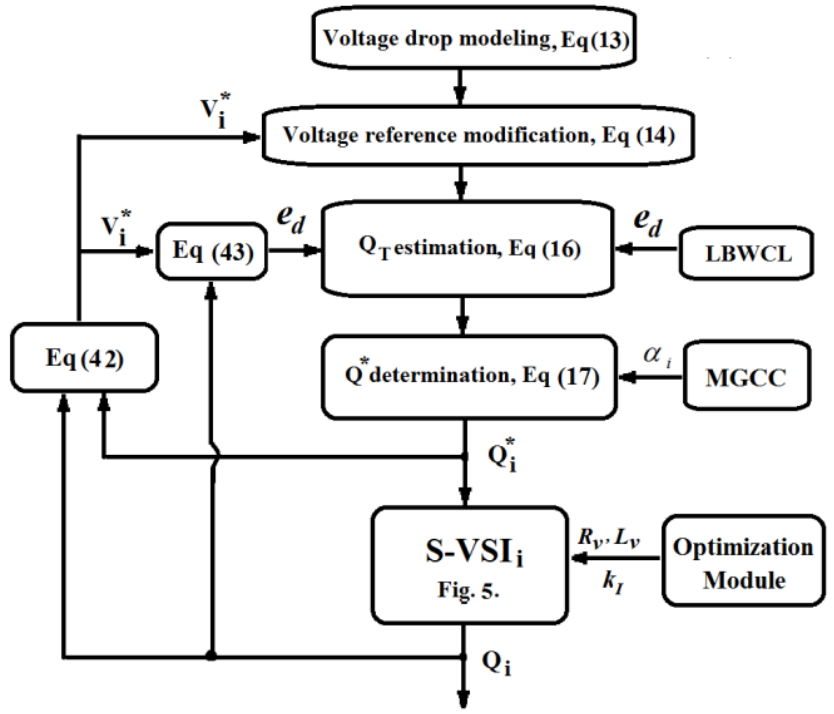

Fig. 7. Flowchart of the proposed control method

\section{Simulation AND Results}

To evaluate the performance of proposed control system, a MG composed of three parallel DG units is simulated in MATLAB $\backslash$ Simulink. The data of DG units is presented in Table I. First, the proposed optimization algorithm is assessed for pole placement. Then the power sharing performance is presented using some case studies.

\section{A. Optimizing S-VSI parameters}

The dominant pole locations of the S-VSI are depicted in Fig. 8. The arrow direction shows the effect of increasing the $k_{I}$ value $\left(k_{I}=0: 1 \mathrm{e}-2\right)$ on the movement of dominant poles; see Fig. 8(a). Increasing integral gain moves $\lambda_{I}$ to the left side of real axis and determines S-VSI performance in reference tracking. However, the integrator gain makes the overall control performance worse $\left(\lambda_{2,3,4}\right)$. The effect of increasing virtual impedance $\left(L_{v}=0: 5 \mathrm{e}-4\right)$ is depicted in Fig. 8(b), while the integrator gain is zero. Increasing virtual impedance has an inverse effect on the coupled poles $\lambda_{2,3}$ and $\lambda_{4}$ in comparison to the integrator gain effect in Fig. 8(a). Fig. 8(c) shows that adopting both virtual impedance and integrator gain results in better control performance while the reference tracking goal is achieved as well. So fuzzy logic is adopted to help PSO to implement a compromise in determining the integrator gain and virtual impedance. It is worth noting that according to Fig. 8(a), selecting a constant optimal value for $k_{I}$ may secure the stability of the control system at different operating points. However, reaching a suitable dynamic performance needs the control parameters be updated in the steady states.

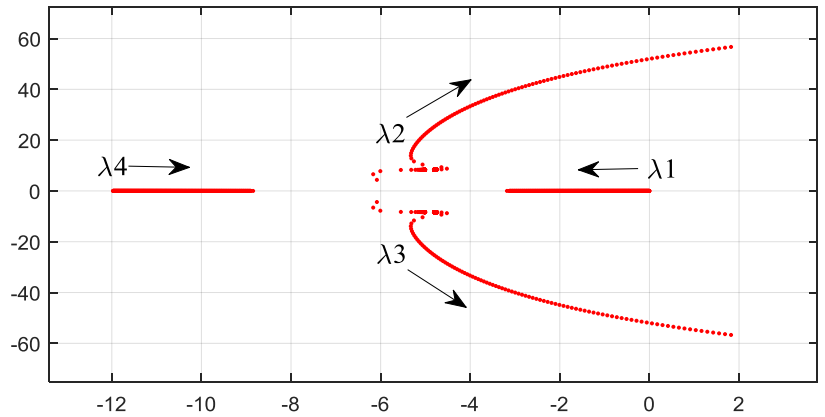

(a)

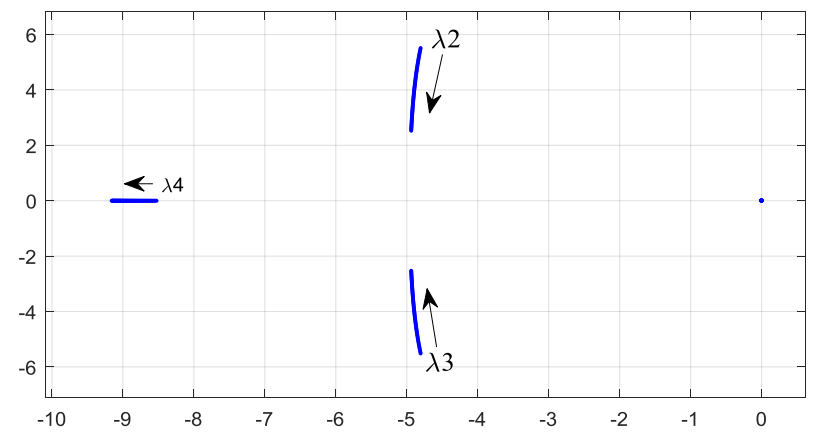

(b)

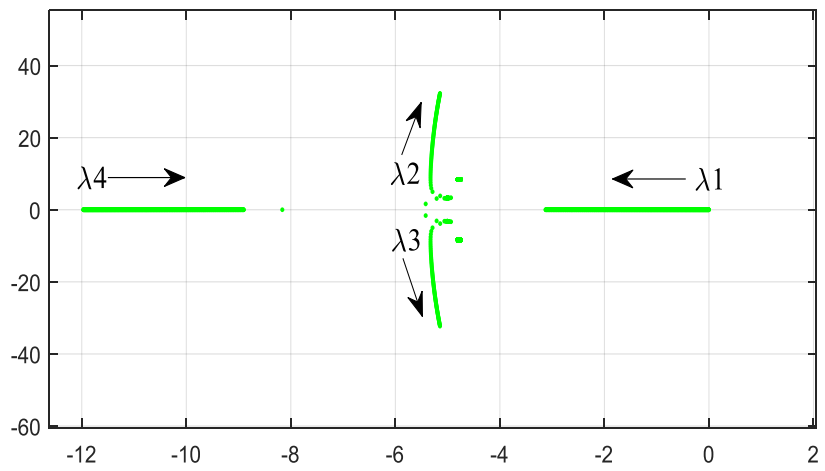

(c)

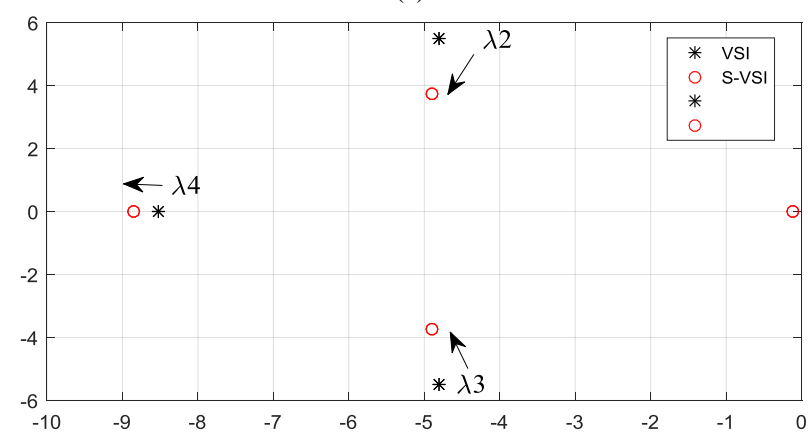

Fig. 8. Dominant pole locus, (a) increasing integrator gain while virtual impedance is not employed, (b) increasing virtual impedance while integrator gain is zero, (c) increasing integrator gain while virtual impedance is adopted, (d) comparison between VSI and optimal S-VSI. 
F-PSO: PSO attempts to move poles to the left hand side of imaginary axis and fuzzy logic is employed to improve the reference tracking response of S-VSI. The inputs of fuzzy logic are the absolute value of $\lambda_{l}$ and the un-damped natural frequency of $\lambda_{2,3}$. The outputs are the updated values of virtual impedance and integrator gain obtained from PSO. The fuzzy rule table for Mamdani fuzzy inference system is given in Table II. A comparison is made between the conventional VSI and optimized S-VSI in terms of the dominant eigenvalue location in Fig. 8(d) $\left(k_{l}=9.12 \mathrm{e}-5\right.$, $\left.L_{v}=2.24 \mathrm{e}-4\right)$, which reveals that an improved dynamic performance is achieved by using the proposed optimal SVSI.

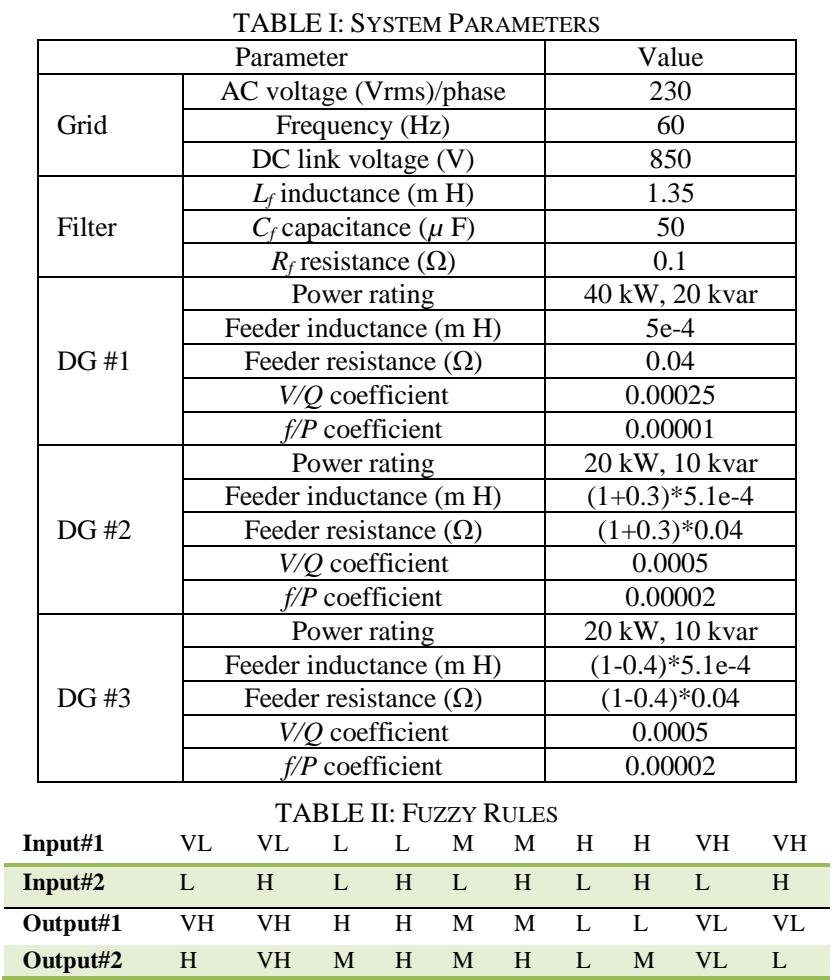

The abbreviation assigned to fuzzy set terms are: Very Low (VL), Low (L), Medium (M), High (H) and Very High (VH).

Computing time: The convergence of PSO in optimizing OF1 is depicted in Fig. 9. With 500 particles and 50 iterations, the convergence is achieved in 20 seconds with a normal laptop computer (processor $1.7 \mathrm{GHz}$ and RAM 6GB). As the optimization process is implemented in the steady state, the computational time $(20 \mathrm{sec})$ with several times repetition $(1 \mathrm{~min})$ to ensure the optimized value is acceptable.

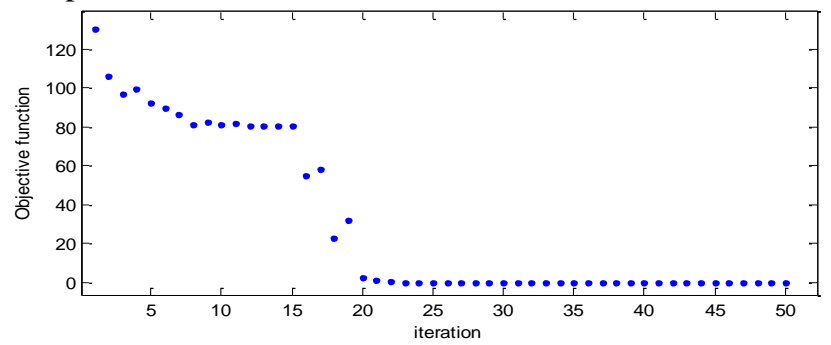

Fig. 9. PSO convergence

\section{B. Power Sharing in Different Scenarios}

The simulated MG topology is depicted in Fig. 10, which is a circuit-based model in Simscape toolbox. In Cases 1-4, circuit breaker (CB) 1 is closed and CB 2 is open. In this way, DG units are connected to $\mathrm{PCC}$ in parallel via different feeder impedances. In Case 5, CB 2 is closed and CB 1 is open to evaluate the effectiveness of the S-VSI in a different MG configuration.

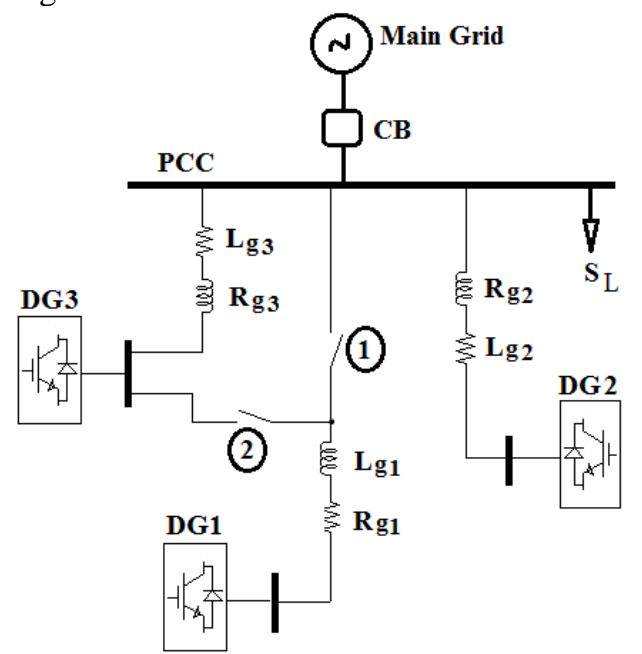

Fig. 10. Simulated MG topology

Case 1: In this case, the MG is operated in IM. DGs 1,2 $\& 3$ are considered as dispatch-able units to supply for load variations. The optimized values of virtual impedance and integrator gain are given in Table III. The simulation results are depicted in Fig. 11. Figs. 11(a)-(b) show the active and reactive power sharing with conventional droop controller. Although the active power sharing is implemented well among DG units, the reactive power is not shared properly among converters because of the feeder impedance mismatch. Especially in Fig. 11(b), when the load increases at $\mathrm{t}=3 \mathrm{sec}$ to the nominal value, VSI 3 is imposed to the overload condition with delivering $Q=15000$ var, which would activate the overload protection relay (this is not included in the simulations). Disconnection of DG3 may lead to the cascading failure of other DG units cascading and finally the MG's collapse. Figs. 11(c)-(d) show the simulation results of the proposed control strategy. As shown, adopting S-VSI is effective in implementing accurate reactive power sharing, and its response speed is very fast, in 0.2 second, even faster than the active power sharing. In order to emphasize the importance of parameter optimization, its effect on the dynamic performance of control system is considered as shown in Fig. 10(e). The integrator gain of S-VSI 1 is $k_{I}=5 \mathrm{e}-4$ which is not optimized. In comparison with Fig. 11(d) with optimized values, the dynamic performance of the control system is not acceptable especially with DG1 going to the overload condition. The voltage magnitude at PCC for the conventional VSI and proposed S-VSI is compared in Fig. 11(f), which reveals that the proposed method improves the power quality as well.

TABLE III: OPTIMAL PARAMETERS FOR CASES 1-4

\begin{tabular}{|l|c|c|c|}
\hline DG & $k_{I}$ & $L_{v}$ & $R_{v}$ \\
\hline DG1 & $1.15 \mathrm{e}-5$ & $3.54 \mathrm{e}-4$ & 0 \\
\hline DG2 & $8.12 \mathrm{e}-5$ & $2.28 \mathrm{e}-4$ & 0 \\
\hline DG3 & $8.04 \mathrm{e}-5$ & $2.25 \mathrm{e}-4$ & 0 \\
\hline
\end{tabular}




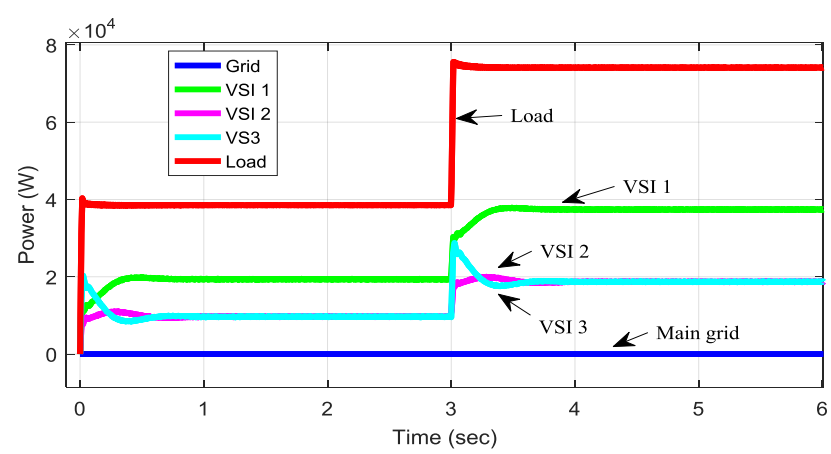

(a)

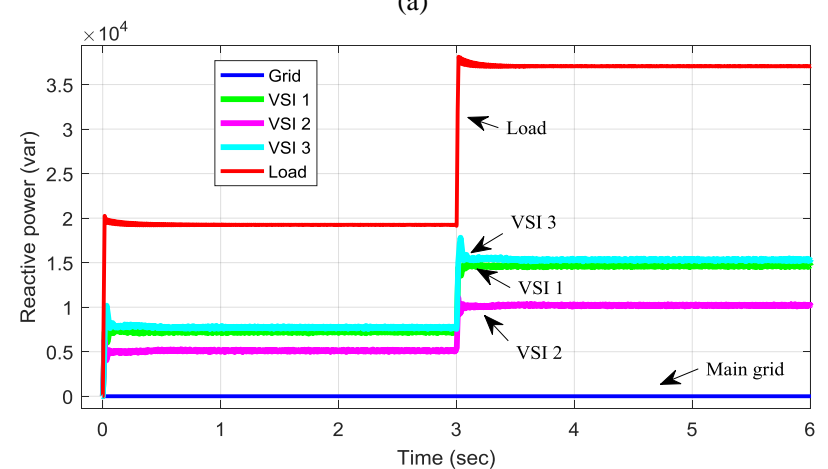

(b)

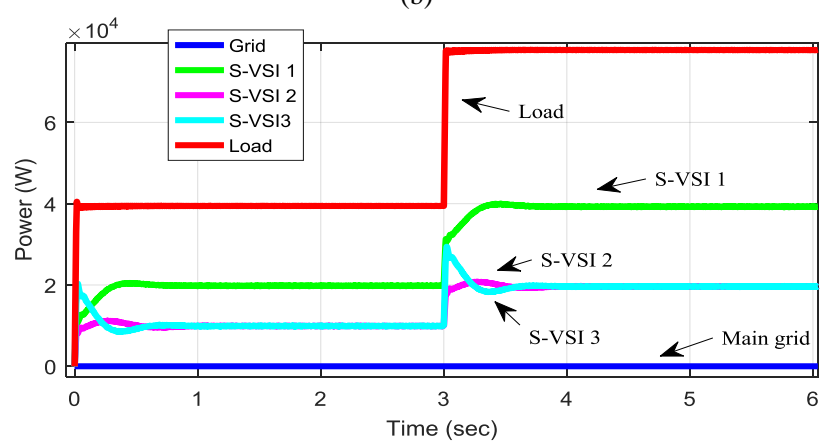

(c)

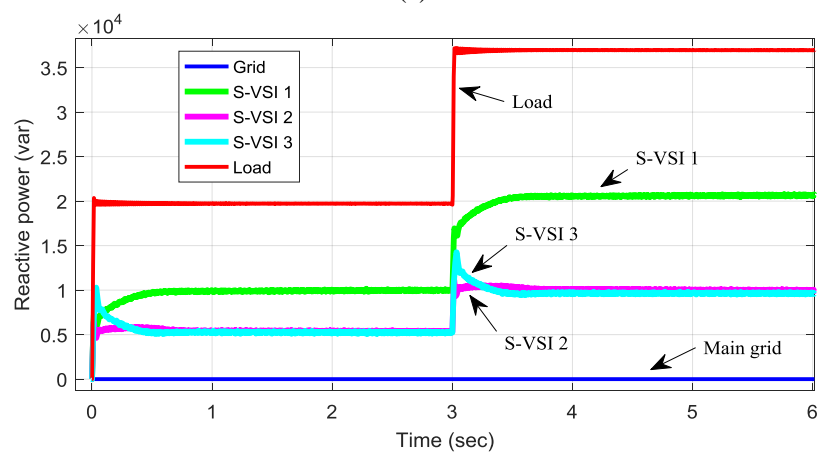

(d)

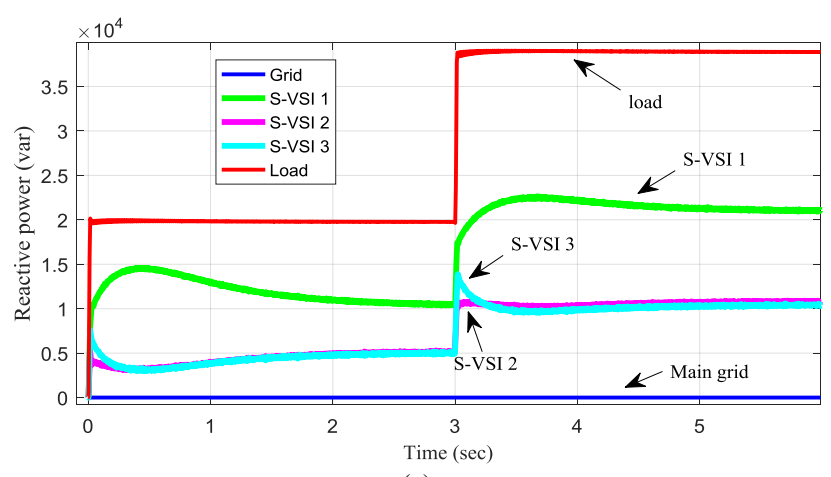

(e)

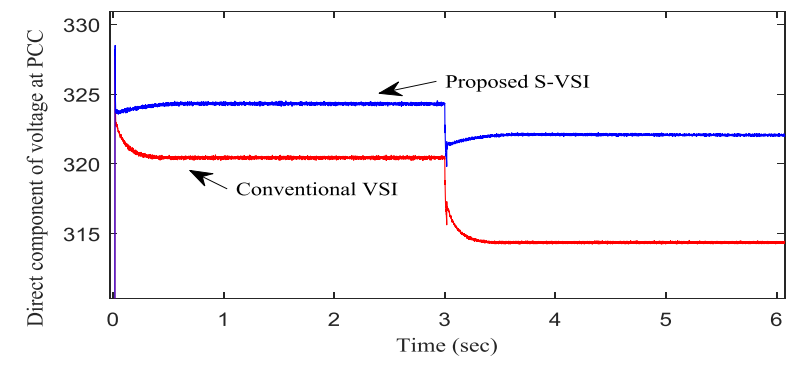

(f)

Fig. 11. Power sharing among DG units in IM, (a), (b) by conventional droop control, (c), (d) by proposed control strategy with optimum parameters, (e) by proposed control strategy without optimum parameters, (f) voltage magnitude at PCC.

Case 2: In this case, the effectiveness of the proposed method in transition from CM to IM is evaluated. The reactive power reference values assigned to DGs $1,2 \& 3$ are $Q^{*}{ }_{1}=10000$ var, $Q^{*}{ }_{2,3}=5000$ var. Fig. 12 (a) shows that VSIs are unable to deliver the required reactive power in $\mathrm{CM}$ and in transition to IM when the MG is disconnected at time $t=2$ sec. It is seen that the reactive power is not shared among DG units according to the droop gains and DG 3 is overloaded. The performance of S-VSIs in terms of reference tracking in $\mathrm{CM}$ and reactive power sharing in IM is depicted in Fig. 12(b). S-VSIs follow the set points well and the reactive power is shared among them appropriately.

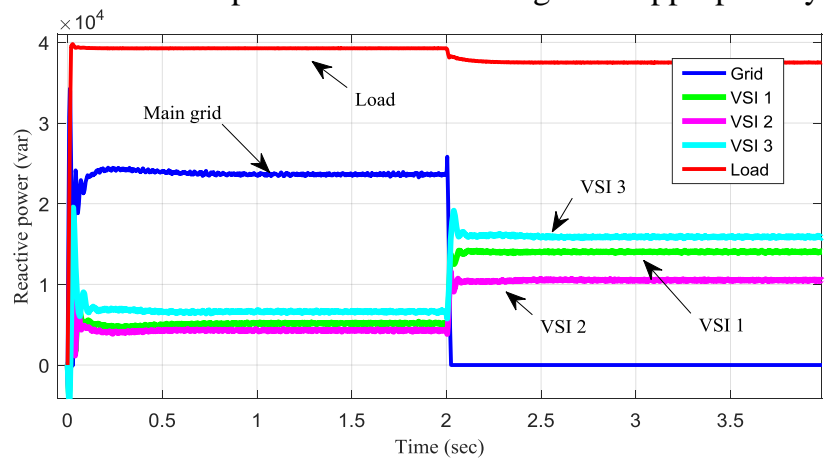

(a)

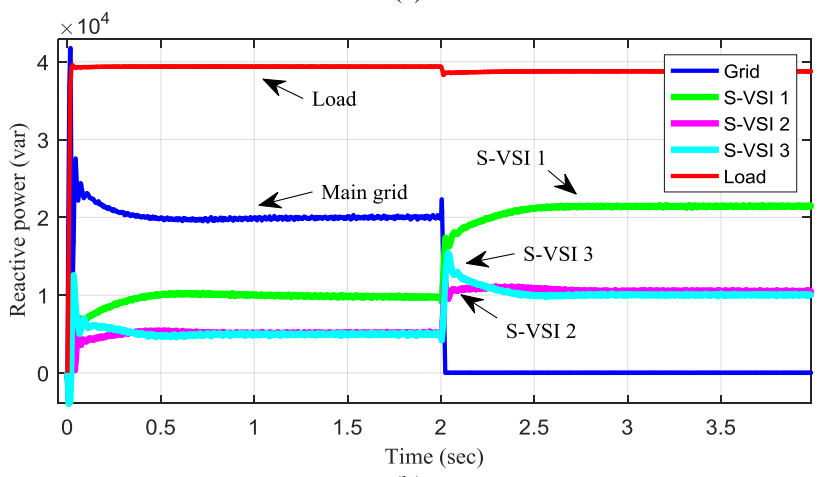

(b)

Fig. 12. Reactive power sharing among DG units in CM and transition to IM, (a) by conventional droop control, (b) by proposed control strategy.

Case 3: In this case, in order to provide the same condition for all DG units, the contribution of all three DG units are considered same by putting droop gains equal to 0.0005. The results for operation in IM is depicted in Fig. 13. The desired performance is achieved by using the proposed control system, while the conventional VSIs are unable to deliver equal reactive power. The other important responsibility of the control system in reactive power 
sharing is to prevent the circulating current between converters. To assess the S-VSI performance in this case, the reactive load is considered zero. Each DG unit should supply the reactive power related to the corresponding feeder inductance. DG 3 with the lowest feeder inductance must deliver the lowest reactive power. Fig. 14(a) reveals the insufficiency of conventional VSI in voltage regulation as there is circulating reactive current among DG units. At $t=2 \mathrm{sec}$, the active power increases and the circulating reactive power increases consequently. On the other hand, the circulating reactive current is limited by using the proposed method and each S-VSI delivers the reactive power according to its feeder inductance; see Fig. 14(b).

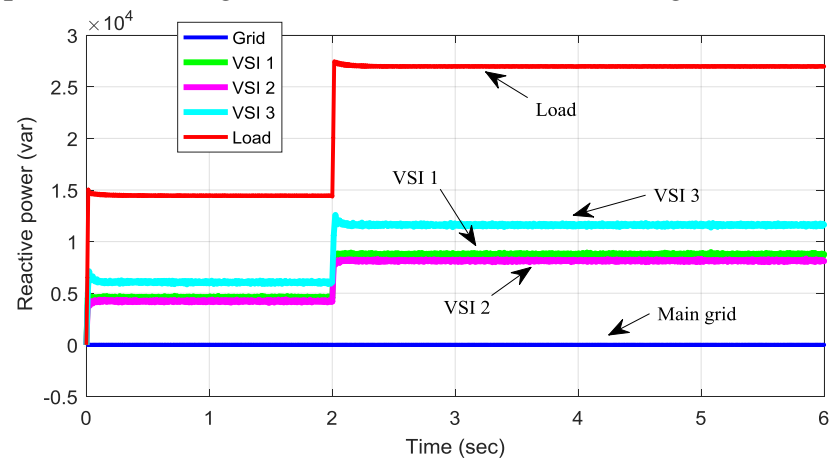

(a)

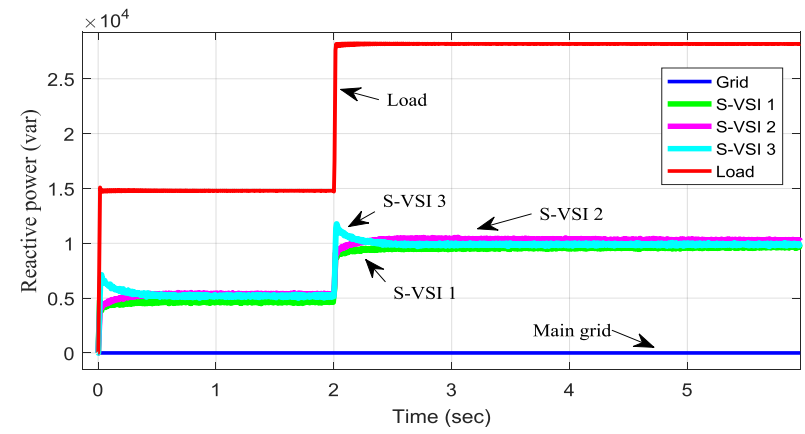

(b)

Fig. 13. Power sharing among DG units in IM, (a) by conventional droop control, (b) by proposed control strategy,

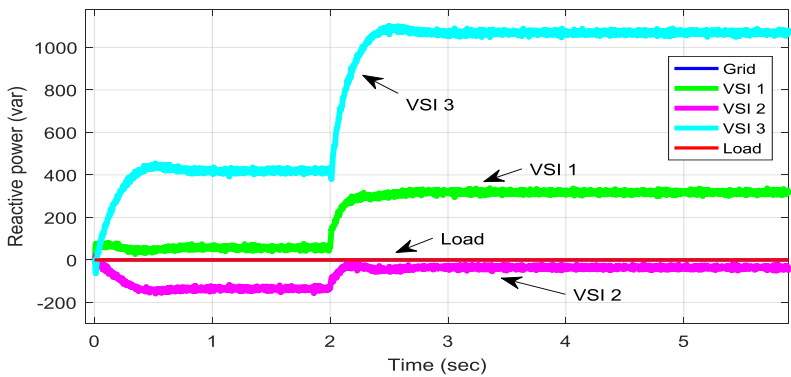

(a)

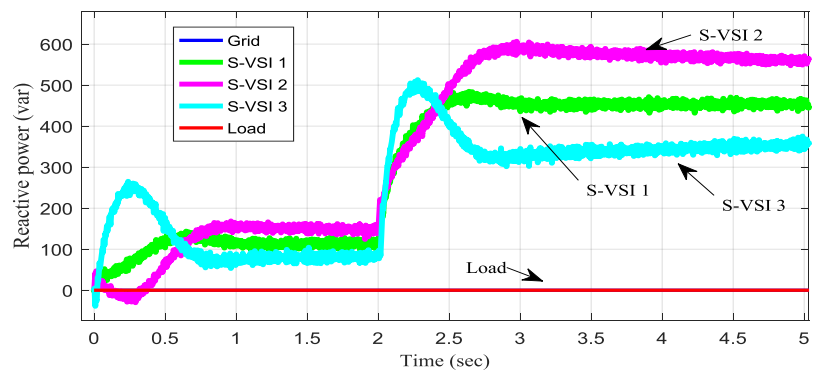

(b)

Fig. 14. Circulating current between DG units in IM, (a) by conventional droop control, (b) by proposed control strategy,
Case 4: In this case, it is assumed that LPBWCL is unavailable, and (43) is used to obtain the MG's bus voltage $e_{d}$. The MG operates in IM. As depicted in Fig. 15, the LPBWCL failure does not influence the performance of control system and the proposed control system could be considered as a kind of fully decentralized control system.

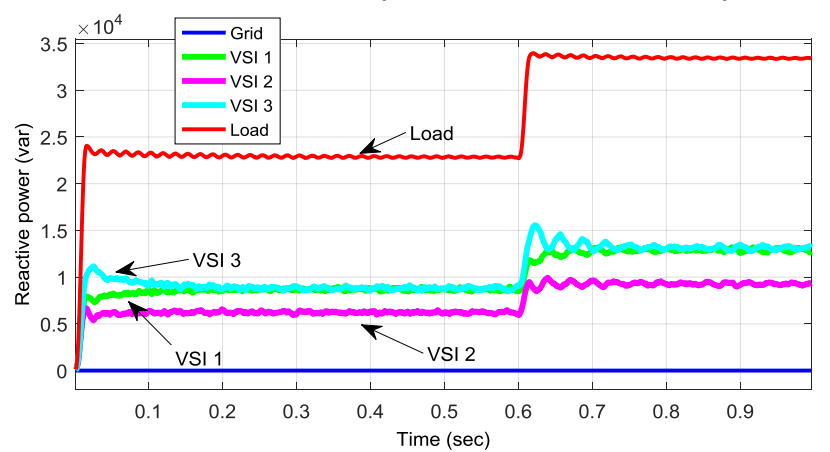

(a)

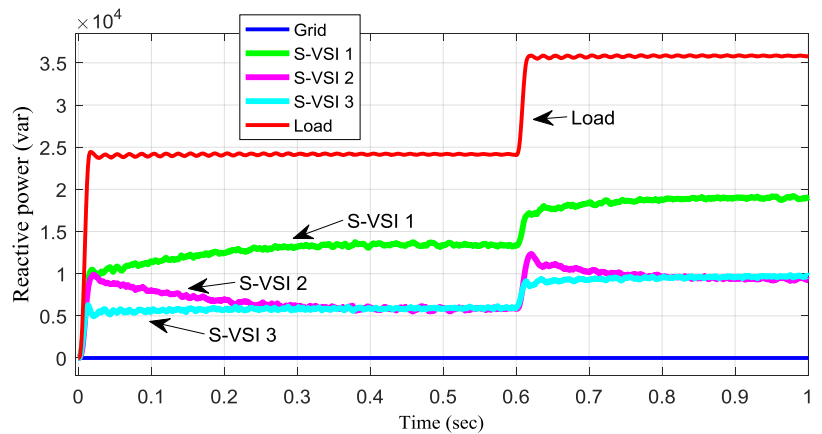

(b)

Fig. 15. Reactive power sharing between DG1, 2 \& 3 in IM, (a) without proposed control system, (b) with proposed control system while LPBWCL is unavailable.

Case 5: In this case, the proposed method is assessed in a different MG configuration where two DG units are located at the same feeder and connected to PCC in series (In Fig. 10, CB 2 is closed while CB 1 is open). The overall performance of the proposed control method is same, and only some consideration should be taken into account:

a) As DGs $3 \& 1$ feed the load through feeder 3 , in the voltage drop modeling in (13)-(14), $(Q 1+Q 3)$ and $(P 1+P 3)$ should be applied for DG 3 . It is easily available as the output power of converter is measured at the output feeders. In this way, equation (15) related to DG 3 estimates the delivered reactive power $(Q 1+Q 3)$ by feeder 3 .

b) For DGs $2 \& 3$, it is not needed to estimate the output reactive power of DG 1 , as is estimated by (15) and $Q 1$ is available by the direct measurement at bus 3.

c) For DG 1, it is still needed to estimate $Q 2$ and $Q 3$. So the direct component of voltage at $\mathrm{PCC}\left(e_{d}\right)$ is required to be sent to this unit, as $Q 3$ is not available for $\mathrm{DG} 1$ to estimate $e_{d}$. However, the existing LBWCL is enough to send this DC value to DG 1.

This process is applicable for $n$ DG units connected in series. The simulation results for this case is shown in Fig. 16. The droop gains for all DG units are asigned 
same which are equal to 0.0005 . The optimal parameters in this case are given in Table IV.

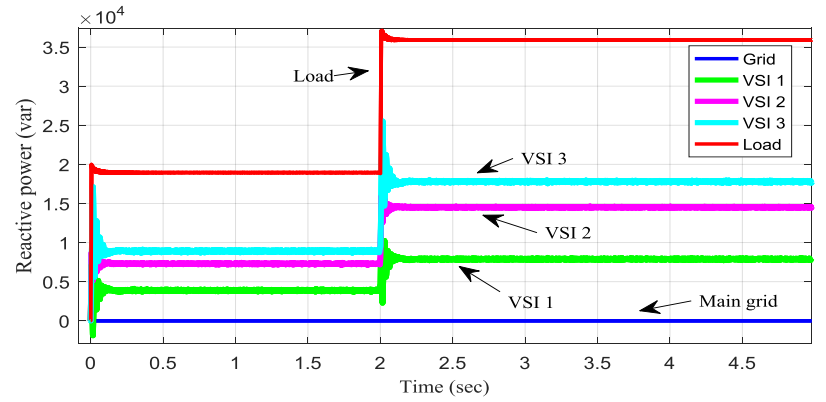

(a)

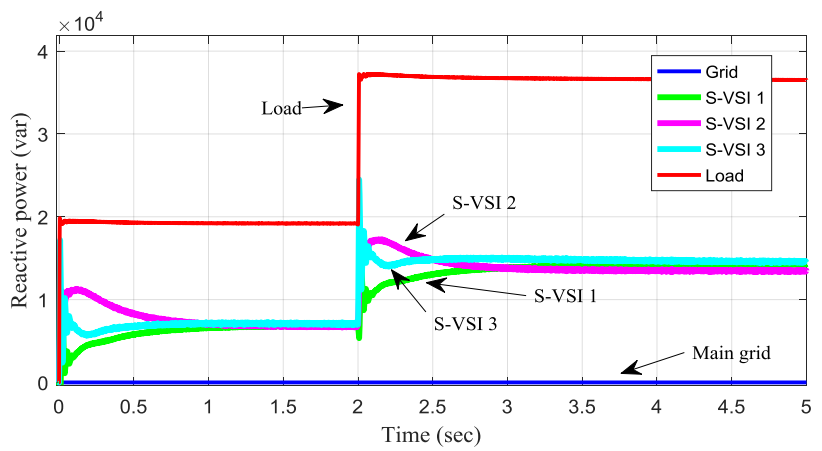

(b)

Fig. 16. Power sharing among DG units in Case 5, (a) by conventional droop control, (b) by proposed control strategy,

TABLE IV: OPTIMAL PARAMETERS FOR CASE 5

\begin{tabular}{|l|c|c|c|}
\hline DG & $k_{I}$ & $L_{v}$ & $R_{v}$ \\
\hline DG1 & $9.35 \mathrm{e}-5$ & $1.22 \mathrm{e}-5$ & 0 \\
\hline DG2 & $8.12 \mathrm{e}-4$ & $4.30 \mathrm{e}-4$ & 0 \\
\hline DG3 & $9.04 \mathrm{e}-5$ & $3.41 \mathrm{e}-5$ & 0 \\
\hline
\end{tabular}

\section{CONCLUSION}

In this paper, a decentralized control strategy with fast response is proposed to address the reactive power sharing issue in MGs. A servo system-based voltage source inverter ( $\mathrm{S}-\mathrm{VSI}$ ) is designed as the reference tracker. A novel method for determining the reactive power set point in IM is presented for each S-VSI, so that each dispatch-able DG unit performs its contribution in reactive power sharing. Virtual impedance is embedded into S-VSI as feedback gain in order to improve the stability as well as decoupling the active and reactive power control. The feedforward integrator gain is designed along with the virtual impedance so that the desired (fast) reference tracking response can be achieved. This adjustment is considered as an optimization problem, and fuzzy PSO is proposed to place S-VSI poles to the desired locations.

MATLAB/Simulink software is used to verify the proposed control system by simulating some scenarios. In these scenarios different MG configurations including DG units with different ratings are considered to prove the effectiveness of the control strategy. Furthermore, despite of removing the existing low band-width communication link (LBWCL), the reactive power is shared fast and accurately, which demonstrates that the proposed control strategy could be considered as a decentralized control system and its reliability is not affected by interruption of LBWCL.

\section{APPENDIX}

The first state variable is defined as:

$$
\dot{\delta}=w_{\text {ref }}
$$

where $\delta$ is the phase angle of converter's output voltage, $w_{\text {ref }}$ is obtained from (1). The average $P$ and $Q$ is achieved after passing instantaneous $p$ and $q$ through a first-order filter. After linearization we have:

$$
\begin{aligned}
& \dot{P}=\left(-w_{c} \cdot P+w_{c} \cdot v_{o_{d_{0}}} \cdot \Delta i_{o_{d}}+w_{c} \cdot i_{o_{d_{0}}} \cdot \Delta v_{o_{d}}+w_{c} \cdot v_{o_{q_{0}}} \cdot \Delta i_{o_{q}}+w_{c} \cdot i_{q_{q_{0}}} \cdot \Delta v_{o_{q}}\right) \\
& \dot{Q}=\left(-w_{c} \cdot Q-w_{c} \cdot v_{o_{d_{0}}} \cdot \Delta i_{o_{q}}-w_{c} \cdot i_{o_{q_{0}}} \cdot \Delta v_{o_{d}}+w_{c} \cdot v_{o_{q_{0}}} \cdot \Delta i_{o_{d}}+w_{c} \cdot i_{o_{d_{0}}} \cdot \Delta v_{o_{q}}\right)
\end{aligned}
$$

where $w_{c}$ is the filter cutting frequency, $v_{o, d q}$ and $i_{o, d q}$ are the $d-q$ components of converter output voltage and current, respectively.

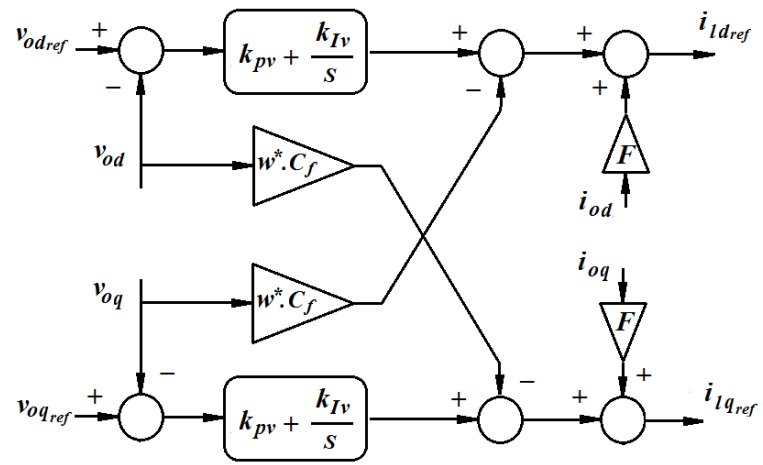

(a)

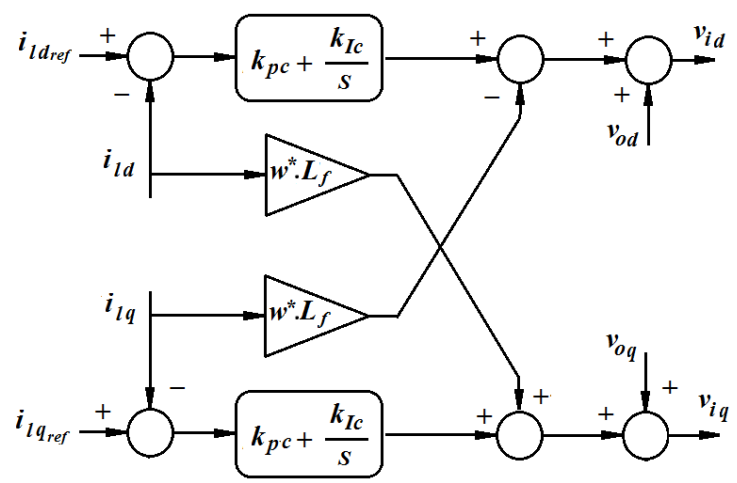

(b)

Fig. 17. VSI inner control loops, (a) voltage control loop, (b) current control loop.

For the VSI's outer (voltage) loop, Fig. 17(a), we have:

$\dot{\varphi}_{d}=v_{\text {od, ref }}-v_{\text {od }} \quad \& \quad \dot{\varphi}_{q}=v_{\text {oq, ref }}-v_{o q}$

where $\varphi_{d, q}$ are the defined state variable for the voltage controller of VSI, $v_{\text {od,ref }}$ comes from (2), and $v_{\text {oq, ref }}$ is equal to zero. The output of VSI's outer (voltage) control loop is the reference current for the inner (current) control loop. For current control loop, Fig. 17(b), we have:

$i_{l d_{r e f}}=F \cdot i_{o d}-w^{*} \cdot C_{f} \cdot v_{o q}+k_{p v} \cdot \dot{\varphi}_{d}+k_{I v} \cdot \varphi_{d}$
$i_{l q_{r e f}}=F \cdot i_{o q}+w^{*} \cdot C_{f} \cdot v_{o d}+k_{p v} \cdot \dot{\varphi}_{q}+k_{I v} \cdot \varphi_{q}$

where $i_{l d, r e f}$ and $i_{l q, r e f}$ are the references for $d$ - $q$ components of converter's output LC filter current, $w^{*}$ is defined in (1), $C_{f}$ is the capacitance of converter's output LC filter, $k_{p v} \& k_{i v}$ are the proportional and integral gains of voltage PI controller, and $F$ is the feedforward gain of converter's output current to improve the controller performance. For the VSI's inner (current) control loop, we have: 


$$
\dot{\gamma}_{d}=i_{l d_{r e f}}-i_{l d} \quad \& \quad \dot{\gamma}_{q}=i_{l q_{r e f}}-i_{l q}
$$

where $\gamma_{d}$ and $\gamma_{q}$ are the defined state variables for the current controller of VSI. The output of current controller is the input voltage reference to the pulse-width modulation module of VSI as:

$$
\begin{aligned}
& v_{i d_{r e f}}=-w^{*} \cdot L_{f} \cdot i_{l_{q}}+k_{p c} \cdot \dot{\gamma}_{d}+k_{I c} \cdot \gamma_{d} \\
& v_{i q_{r f}}=w^{*} \cdot L_{f} \cdot i_{l_{d}}+k_{p c} \cdot \dot{\gamma}_{q}+k_{I c} \cdot \gamma_{q}
\end{aligned}
$$

where $L_{f}$ is the inductance of converter's output LC filter, $k_{p v}$ $\& k_{i v}$ are the proportional and integral gains of the PI controller in the current control loop. Considering the converter as an ideal VSI which transfers the reference voltage to its output, for the inductance current of LC filter, we have:

$$
\begin{aligned}
& \dot{i_{l_{d}}}=-\frac{R_{f}}{L_{f}} i_{l_{d}}+w_{r e f} . i_{l_{q}}+\frac{1}{L_{f}} v_{i_{d}}-\frac{1}{L_{f}} v_{o_{d}} \\
& \dot{i_{l q}}=-\frac{R_{f}}{L_{f}} i_{l_{q}}-w_{r e f} . i_{l_{d}}+\frac{1}{L_{f}} v_{i_{q}}-\frac{1}{L_{f}} v_{o_{q}}
\end{aligned}
$$

where $R_{f}$ is the resistance of LC filter. For the LC filter capacitance voltage, we have:

$$
\begin{aligned}
& \dot{v}_{o_{d}}=w_{r e f} \cdot v_{o_{q}}+\frac{1}{C_{f}} i_{l_{d}}-\frac{1}{C_{f}} i_{o_{d}} \\
& \bullet_{o_{q}}=-w_{r e f} \cdot v_{o_{d}}+\frac{1}{C_{f}} i_{l_{q}}-\frac{1}{C_{f}} i_{o_{q}}
\end{aligned}
$$

Finally, for the converter's output current, we have:

$$
\begin{aligned}
& \dot{i_{o_{d}}}=-\frac{R_{g}}{L_{g}} i_{o_{d}}+w_{r e f} . i_{o_{q}}+\frac{1}{L_{g}} v_{o_{d}}-\frac{1}{L_{g}} e_{d}^{\prime} \\
& \dot{i_{o_{q}}}=-\frac{R_{g}}{L_{g}} i_{o_{q}}-w_{r e f} . i_{o_{d}}+\frac{1}{L_{g}} v_{o_{q}}-\frac{1}{L_{g}} e_{q}^{\prime}
\end{aligned}
$$

where $R_{g}$ and $L_{g}$ are the resistance and inductance of interconnecting grid feeder, respectively; $e_{d}^{\prime}$ and $e_{q}^{\prime}$ are the $d-q$ components of MG bus voltage which is transferred to the VSI frequency reference frame $(\delta)$ by the transformation matrix as:

$$
\left[\begin{array}{l}
e_{d}^{\prime} \\
e_{q}^{\prime}
\end{array}\right]=\left[\begin{array}{cc}
\cos \left(\delta_{B}-\delta\right) & -\sin \left(\delta_{B}-\delta\right) \\
\sin \left(\delta_{B}-\delta\right) & \cos (\delta-\delta)
\end{array}\right]\left[\begin{array}{l}
e_{d} \\
e_{q}
\end{array}\right]
$$

Here $e_{d}$ and $e_{q}$ are the $d$-q components of MG bus voltage and $\delta_{B}$ is the phase angle of MG bus voltage.

\section{References}

[1] M. H. Moradi, M. Eskandari, S. M. Hosseinian, "Operational Strategy Optimization in an Optimal Sized Smart Microgrid", IEEE Trans. Smart Grid, vol. 6, no. 3, pp. 1087-1095, May. 2015.

[2] M. H. Moradi, M. Eskandari, S. M. Hosseinian, "Cooperative control strategy of energy storage systems and micro sources for stabilizing microgrids in different operation modes," International Journal of Electrical Power \& Energy Systems, vol. 6, pp. 390-400, June. 2016.

[3] M. H. Moradi, M. Eskandari, "A Hybrid method for Simultaneous optimization of DG capacity and Operational Strategy in Microgrids considering uncertainty in electricity price forecasting," Renewable Energy 2014, 68, $697-714$.

[4] N. Pogaku, M. Prodanovic', T. C. Green, "Modeling, Analysis and Testing of Autonomous Operation of an Inverter-Based Microgrid," IEEE Trans. Power Electron, vol. 22, no. 2, pp. 613-625, March 2007.

[5] S.Augustine, M. K. Mishra, N. Lakshminarasamma, "Adaptive Droop Control Strategy for Load Sharing and Circulating Current Minimization in Low-Voltage Standalone DC Microgrid," IEEE Trans. Sustainable energy, vol. 6, no. 1, pp. 132-141, Jan 2015.

[6] P. H. Divshali, A. Alimardani, and S. H. Hosseinian, "Decentralized cooperative control strategy of microsources for stabilizing autonomous VSCbased microgrids," IEEE Trans. Power Syst., vol. 24, no. 4, pp. 1949-1959, Nov. 2012.
[7] M. H. Moradi, M. Eskandari, P. Siano, "Safe transition from connection mode to islanding mode in Microgrids", IEEE, Electrical Engineering (ICEE), 2016 24th Iranian Conference on, pp. 1902-1907, May. 2016.

[8] N. R. Tummuru, M. K. Mishra, S. Srinivas, "Multifunctional VSC Controlled Microgrid Using Instantaneous Symmetrical Components Theory," IEEE Trans. Sustainable energy, vol. 5, no. 1, pp. 313-322, Jan 2014.

[9] J. Rocabert, A. Luna, F. Blaabjerg, and P. Rodríguez, "Control of power converters in AC microgrids," IEEE Trans. Power Electron, vol. 27, no. 11, pp. 4734-4749, Nov. 2012.

[10] H. Han, X. Hou, J. Yang, J. Wu, M. Su, J. M. Guerrero, "Review of Power Sharing Control Strategies for Islanding Operation of AC Microgrids," IEEE Trans. Smart Grid, vol. 7, no. 1, pp. 200-215, 2016.

[11] A.Tah, D. Das, "An Enhanced Droop Control Method for Accurate Load Sharing and Voltage Improvement of Isolated and Interconnected DC Microgrids," IEEE Trans. Sustainable energy, vol. 7, no. 3, pp. 1194-1204, Jul 2016.

[12] R.Aghatehrani, R. Kavasseri, "Reactive Power Management of a DFIG Wind System in Microgrids Based on Voltage Sensitivity Analysis," IEEE Trans. Sustainable energy, vol. 2, no. 4, pp. 451-458, Oct 2011.

[13] H. Han, Y. Liu, Y. Sun, M. Su, J. M. Guerrero, "An Improved Droop Control Strategy for Reactive Power Sharing in Islanded Microgrid," IEEE Trans. Power Electron, vol. 30, no. 6, pp. 3133-3141, June. 2015.

[14] X. Sun, Y. Hao, Q. Wu, X. Guo, B. Wang, "A Multifunctional and Wireless Droop Control for Distributed Energy Storage Units in Islanded AC microgrid Applications," IEEE Trans. Power Electronic, Early access article, 2016.

[15] Y. Wang, Z. Chen, X. Wang, Y. Tian, Y. Tan, Ch. Yang, "An EstimatorBased Distributed Voltage-Predictive Control Strategy for AC Islanded Microgrids," IEEE Transactions on Power Electronics, vol. 30, no. 7, pp. 39343951, July. 2015.

[16] H. Mahmood, D. Michaelson, and J. Jiang, "Accurate reactive power sharing in an islanded microgrid using adaptive virtual impedances," IEEE Trans. Power Electron, vol. 30, no. 3, pp. 1605-1617, Mar. 2015.

[17] H. Zhang, S. Kim, Q. Sun, J. Zhou, "Distributed Adaptive Virtual Impedance Control for Accurate Reactive Power Sharing Based on Consensus Control in Microgrids,” IEEE Trans. Smart Grid, vol. 6, no. 3, pp. 1087-1095, May. 2016.

[18] J. Schiffer, T. Seel, J. Raisch, T. Sezi, "Voltage Stability and Reactive Power Sharing in Inverter-Based Microgrids with Consensus-Based Distributed Voltage Control," IEEE Trans. on control system technology, vol. 24, no. 10, pp. 96-109, January. 2016.

[19] J. W. Simpson-Porco, Q. Shafiee, F. D“orfler, J. M. Vasquez, J. M. Guerrero, and F. Bullo, "Secondary frequency and voltage control of islanded microgrids via distributed averaging," IEEE Transactions on Industrial Electronics, vol. 62, no. 11, pp. 7025-7038, 2015.

[20] X. Yang, Y. Du, J. Su, L. Chang, Y. Shi, J. Lai, "An Optimal Secondary Voltage Control Strategy for an Islanded Multi-bus Microgrid," IEEE Trans. Power Electronic, Early access article, 2016.

[21] M. Pulcherio et al., "Evaluation of Control Methods to Prevent Collapse of a Mixed Source Microgrid," IEEE Transactions on Industry Application, Early access article, 2016.

[22] J. He, Y. W. Li, "Analysis, Design, and Implementation of Virtual Impedance for Power Electronics Interfaced Distributed Generation," IEEE Trans. Industry Applications, vol. 47, no. 6, Nov 2011.

[23] J. He, Y. W. Li, J. M. Guerrero, F. Blaabjerg, and J. C. Vasquez, "An Islanding Microgrid Power Sharing Approach Using Enhanced Virtual Impedance Control Scheme," IEEE Transactions on Power Electronics, vol. 28, no. 11, pp. 5272-5282, Nov. 2013.

[24] Y. Zhu, F. Zhuo, F. Wang, B. Liu, R. Gou, Y. Zhao, “A Virtual Impedance Optimization Method for Reactive Power Sharing in Networked Microgrid," IEEE Transactions on Power Electronics, vol. 31, no. 4, pp. 2890-2904, April. 2016.

[25] V. L. Syrmos, C. T. Abdallah, P. Dorato, K. Grigoriadis. "Static output feedback-a survey." Automatica, vol. 33, no. 2, pp. 125-137, 1997.

[26] Y. Cao, J. Lam, Y. Sun. "Static output feedback stabilization: an ILMI approach." Automatica, vol. 34, no. 12, pp. 1641-1645, 1998.

[27] L. E. Ghaoui, F. Oustry, M. AitRami. "A cone complementarity linearization algorithm for static output-feedback and related problems." IEEE transactions on automatic control, vol. 42, no. 8, pp. 1171-1176, Aug 1997. 